\title{
Avaliação do risco cardiovascular em mulheres docentes no fim do período reprodutivo
}

Assessment of the cardiovascular risk in female professors at the end of their reproductive period Evaluación del riesgo cardiovascular en mujeres docentes al final de su período reproductivo

\section{Tamires Tássia dos Santos ${ }^{\mathrm{I}}$, Sofia Louise Santin Barilli ${ }^{\mathrm{II}}$, Mariana Bello Porciuncula ${ }^{\mathrm{III}}$, Rafaela dos Santos Charão de Almeida ${ }^{\mathrm{IV}}$, Andréia Martins Specht ${ }^{\mathrm{V}}$}

\begin{abstract}
Resumo: Objetivo: avaliar o risco cardiovascular em mulheres docentes do ensino superior no fim do período reprodutivo. Método: estudo quantitativo, transversal, realizado em universidade privada do Sul do Brasil, em setembro de 2018. Incluíram-se docentes no climatério ou menopausa (idade superior a 44 anos, com dosagem de colesterol no último ano e que conhecessem sua pressão arterial sistólica). Dados sociodemográficos e clínicos foram coletados a partir de questionário estruturado; o risco cardiovascular (RCV) foi mensurado por meio do escore de Framingham. Resultados: houve diferença no RCV entre as fases (climatério e menopausa). Todas as mulheres climatéricas apresentaram RCV baixo; já as mulheres na menopausa apresentaram RCV baixo (73\%), RCV intermediário $(24,7 \%)$ e RCV alto $(2,2 \%)$. Conclusão: existe relação significativa entre menopausa e aumento do $\mathrm{RCV}$, justificando ser mais um fator de risco para as mulheres.
\end{abstract}

Descritores: Fatores de Risco; Menopausa; Doenças Cardiovasculares; Mulheres; Enfermagem

Abstract: Objective: To assess the cardiovascular risk in female professors at the end of their reproductive period. Method: A cross-sectional quantitative study conducted in a private university in southern Brazil in September 2018, including climacteric or post-menopausal women older than 44 years of age who had measured their cholesterol levels in the last year and who knew their systolic blood pressure level. Sociodemographic and clinical data were collected using a structured questionnaire; and the cardiovascular risk (CVR) was measured using

\footnotetext{
I Enfermeira. Graduada em Enfermagem. Universidade do Vale do Rio dos Sinos. São Leopoldo, Rio Grande do Sul, Brasil. tamirests.santos@gmail.com. https://orcid.org/0000-0001-9394-4606

II Enfermeira. Mestre em Enfermagem. Universidade do Vale do Rio dos Sinos, Hospital Nossa Senhora da Conceição/GHC. Porto Alegre, Rio Grande do Sul, Brasil. sofiabarilli@gmail.com. https://orcid.org/0000-0002-8635-6029

III Enfermeira. Doutora em Enfermagem. Universidade do Vale do Rio dos Sinos. Porto Alegre, Rio Grande do Sul, Brasil. maribellinho@gmail.com. https://orcid.org/0000-0002-0082-2763

Iv Enfermeira. Mestranda em Avaliação de Tecnologias para o Sistema Único de Saúde - Grupo Hospitalar Conceição. Hospital Nossa Senhora da Conceição/GHC. Porto Alegre, Rio Grande do Sul, Brasil. rafacharao@gmail.com. https://orcid.org/0000-0002-5003-2284

v Enfermeira. Doutora em Enfermagem. Programa de Pós-graduação em Enfermagem Universidade do Vale do Rio dos Sinos, Hospital Nossa Senhora da Conceição/GHC. Porto Alegre, Rio Grande do Sul, Brasil. spechtandreia@gmail.com. https://orcid.org/0000-0001-8997-3279
} 
Avaliação do risco cardiovascular em mulheres docentes no fim do período reprodutivo | 2

the Framingham risk score. Results: There was a difference in the CVR between the phases (climacteric and menopause). All the climacteric women had a low CVR, whereas post-menopausal women were classified into low (73\%), intermediate (24.7\%), and high (2.2\%) CVR risk. Conclusion: There was a significant relationship between menopause and increased CVR, justifying the fact that this is yet another risk factor for women.

Keywords: Risk Factors; menopause; Cardiovascular Diseases; Women; Nursing

Resumen: Objetivo: evaluar el riesgo cardiovascular en mujeres docentes de educación superior que se encuentran en el final de su período reproductivo. Método: estudio cuantitativo y transversal realizado en una universidad privada del sur de Brasil durante el mes de septiembre de 2018. Se incluyó a docentes que se encontraban en el climaterio o en la menopausia (más de 44 años, con medición de colesterol en el último año, y que conociesen su presión arterial sistólica). Se recopilaron datos sociodemográficos y clínicos a partir de un cuestionario estructurado; el riesgo cardiovascular (RCV) se evaluó por medio del puntaje de Framingham. Resultados: se registró una diferencia en el RCV entre las fases (climaterio y menopausia). Todas las mujeres en el período del climaterio presentaron un RCV bajo, mientras que las mujeres entradas en la menopausia presentaron RCV bajo (73\%), intermedio $(24,7 \%)$ y alto $(2,2 \%)$. Conclusión: existe una relación significativa entre la menopausia y un aumento en el RCV, lo que justifica considerarlo como un factor de riesgo adicional para las mujeres.

Descriptores: Factores de riesgo; Menopausia; Enfermedades cardiovasculares; Mujeres; Enfermería

\section{Introdução}

As doenças cardiovasculares (DCV) são a principal causa de óbito no mundo, tendo sido responsáveis por 17,7 milhões de óbitos no ano de 2015 , o que representa $31 \%$ de todas as mortes. ${ }^{1}$ Além disso, quando realizada a estratificação por sexo, as DCV lideram as estatísticas de falecimento de mulheres. Nos Estados Unidos da América, destacam-se como a principal causa de mortalidade no gênero feminino, responsáveis por aproximadamente uma em cada cinco mortes femininas no ano de $2017 .{ }^{2}$ Ainda, é possível observar que a incidência aumenta na fase de declínio dos hormônios ovarianos, caracterizada de forma fisiológica pela chegada da menopausa. ${ }^{3}$

O fim do período do ciclo reprodutivo feminino é determinado pelas fases de climatério e menopausa. O climatério é quando se iniciam os primeiros sintomas marcados por alterações dos ciclos menstruais, terminando quando a menopausa se inicia, sendo esta determinada pela cessação da menstruação por doze meses contínuos. A idade de início deste processo varia, 
ocorrendo em média aos 51 anos. Nesta fase, as mulheres têm aumentado o risco de desenvolver DCV. ${ }^{4}$ A transição para a fase da menopausa é marcada pela diminuição dos hormônios ovarianos - tanto estrógenos, quanto testosterona - os quais desempenham papel importante na função endotelial, no tônus vascular e também na função cardíaca, fazendo com que cresça o risco cardiovascular $(\mathrm{RCV})$ no período pós-menopausa. Nesse período, destacam-se a presença de alguns fatores de risco como: a queda das lipoproteínas de alta densidade (HDLs), o aumento das lipoproteínas de baixa densidade (LDLs), bem como as alterações no metabolismo e na pressão arterial. ${ }^{5}$ Até 2030, estima-se que a população brasileira feminina seja superior a 113 milhões. ${ }^{6}$ Torna-se possível extrapolar esse dado, com a previsão de que nesse mesmo ano, aproximadamente $21 \%$ das mulheres no Brasil, cerca de 24 milhões, estarão na faixa etária em que ocorre o climatério e a menopausa. ${ }^{6}$

Diante desse contexto, afirma-se que a avaliação do RCV apresenta relevância, ${ }^{7}$ o que é reforçado quando se observa que, no cenário nacional, nos últimos dez anos, as doenças do aparelho circulatório também lideram as causas de óbito, a frente das neoplasias e doenças respiratórias. ${ }^{8}$ A partir da fase do climatério, a incidência das DCV aumenta de forma significativa, o que se justifica pelas mudanças hormonais, que repercutem na disfunção endotelial, apontada como um fator no processo das DCV. ${ }^{9}$

A expectativa de vida do brasileiro passou para 75,8 anos e as mulheres vivem em média mais do que os homens, 79,4 anos e 72,9 anos, respectivamente. ${ }^{10} \mathrm{Na}$ população feminina, as manifestações clínicas das DCV podem aparecer em torno de 10 a 15 anos após a menopausa, o que está associado à proteção vascular fornecida pelo estrogênio. ${ }^{11}$ Considerando o aumento da expectativa de vida entre as mulheres, torna-se interessante que o período da menopausa passe a ser avaliado para que se possa atuar preventivamente.

O Escore de Risco de Framingham (ERF) - um preditor de risco cardiovascular (RCV) - é uma ferramenta que se baseia em variáveis como sexo, idade, pressão arterial sistólica (PAS), 
Avaliação do risco cardiovascular em mulheres docentes no fim do período reprodutivo 4

colesterol total, fração de HDL, presença de diabetes mellitus (DM) e tabagismo. Cada variável avaliada possui valores numéricos correspondentes, específicos para mulheres ou homens. Após a soma, o risco é classificado como baixo $(<10 \%)$, intermediário ou moderado (10 a $20 \%)$ e alto (>20\%). A avaliação de tais fatores permite identificar qual a probabilidade do indivíduo, sem diagnóstico prévio, ter algum evento cardiovascular nos próximos 10 anos de vida, estabelecendo medidas para esta redução. ${ }^{12}$

O conhecimento do RCV em mulheres tem apresentado imprecisões na detecção precoce e consequentemente na prevenção do surgimento das DCV, o que torna significativa a exploração de fatores de risco novos, para que com isso possa haver uma queda nas taxas de morbimortalidade por estas causas. ${ }^{13}$ Considerando o amplo papel da Enfermagem na prevenção de eventos cardiovasculares, é essencial que esses profissionais se apropriem desse conhecimento para que possam desenvolver programas e estratégias de promoção da saúde, objetivando a redução dessas taxas e proporcionando benefícios como melhor qualidade de vida a estas mulheres.

A questão de pesquisa foi: qual o risco cardiovascular em mulheres docentes do ensino superior no fim do período reprodutivo? Tal questão gerou o seguinte objetivo: avaliar o RCV em mulheres docentes do ensino superior no fim do período reprodutivo.

\section{Método}

Trata-se de um estudo transversal, com abordagem quantitativa, desenvolvido em uma universidade privada, na região do Vale dos Sinos, no Sul do Brasil. Foram incluídas todas as docentes, independente da área de atuação (cursos de graduação, pós-graduação e idiomas), que estivessem no período do climatério ou menopausa - com idade acima de 44 anos (idade estabelecida para a inclusão das mulheres na menopausa precoce); que tivessem realizado dosagem de colesterol total e HDL no último ano; e que conhecessem o valor regular da sua 
pressão arterial sistólica. Não fizeram parte da amostra mulheres que possuíam histórico de evento cardiovascular prévio como infarto agudo do miocárdio (IAM), acidente vascular cerebral $(A V C)$ - pois o ERF não se aplica a pacientes com doenças coronarianas diagnosticadas. ${ }^{12}$ No início da pesquisa, as docentes foram informadas quanto à definição das fases - climatério e menopausa - as quais caracterizam o fim do período reprodutivo das mulheres. Considerandose que o total de docentes mulheres com idade acima de 44 anos na universidade é de 213 , a margem de erro de $5 \%$ e grau de confiança de $95 \%$, estimou-se que a amostra deveria ser composta por 138 docentes.

A coleta de dados ocorreu por meio da aplicação de um questionário elaborado pelas autoras com perguntas fechadas, contendo variáveis sociodemográficas e clínicas para caracterização da amostra, além das variáveis necessárias para o cálculo do RCV. O convite para participação no estudo, bem como o formulário de pesquisa, foi encaminhado para o endereço de e-mail institucional das participantes, obtido junto ao departamento de recursos humanos da universidade. Cinco dias após o envio do primeiro e-mail, foi enviada nova mensagem direcionada às docentes que ainda não haviam respondido o questionário reforçando o convite. Após o recebimento das respostas, foi calculado o valor percentual do risco de desenvolver a doença cardiovascular nos dez anos subsequentes, utilizando-se o ERF, com as seguintes categorias: risco baixo $(<10 \%)$, risco intermediário $(10 \%$ a $20 \%)$ e risco alto $(>20 \%) .{ }^{12}$ A coleta foi realizada no mês de setembro de 2018 .

Os dados foram inseridos em um banco de dados, no pacote estatístico Statistical Package for Social Sciences (SPSS) versão 20.0 e analisados por meio de estatística descritiva. Para as variáveis contínuas foram utilizados média e desvio padrão ou mediana e percentis, conforme a distribuição dos dados. As variáveis categóricas foram apresentadas números absolutos e frequências relativas. Para relacionar o risco de desenvolvimento de DCV com variáveis clínicas, foram utilizados os coeficientes de correlação de Pearson ou Sperman, de acordo com 
Avaliação do risco cardiovascular em mulheres docentes no fim do período reprodutivo |6

distribuição dos dados e Qui-Quadrado para associação entre os dados categóricos. Um valor $\mathrm{p}$ $<0,05$ foi considerado significativo.

O projeto foi aprovado quanto aos seus aspectos éticos e metodológicos pelo Comitê de Ética em Pesquisa da Instituição em 21/08/2018 (CAAE: 91756118.2.0000.5344). Foi aplicado Termo de Consentimento Livre e Esclarecido às participantes da pesquisa, que foi conduzida de acordo com as Diretrizes e Normas regulamentadoras de Pesquisa Envolvendo Seres Humanos (Resolução CNS nº 466, de 12 de dezembro de 2012) ${ }^{14}$ e complementares ao Conselho Nacional de Saúde. ${ }^{15}$

\section{Resultados}

Do total das 213 docentes na faixa etária acima de 44 anos de idade, 31 (14,6\%) retornaram o e-mail afirmando que não se encontravam na menopausa e no climatério. Outras 15 docentes (7\%) responderam que não tinham conhecimento dos dados necessários para a pesquisa e 27 docentes $(12,7 \%)$ não retornaram os e-mails. As docentes participantes representaram todas as áreas do conhecimento, compondo uma amostra final de 140 mulheres que estavam na docência no ensino superior, há uma média de tempo de 22,5 anos (Tabela 1).

Tabela 1 - Características sociodemográficas e clínicas da amostra. São Leopoldo, 2018. (n=140)

\begin{tabular}{lc}
\hline Características & $\mathbf{n}(\mathbf{\%})$ \\
\hline Tempo na docência & $22,5 \pm 8,9$ \\
Idade & $56,5 \pm 5,8$ \\
Idade no climatério & $50 \pm 2,9$ \\
Idade na menopausa & $56,5 \pm 3,4$ \\
Climatério & $51(36,4)$ \\
Menopausa & $89(63,6)$ \\
Hipertensão arterial sistêmica em tratamento & $24(17,1)$ \\
\hline
\end{tabular}


7 | Santos TT, Barilli SLS, Porciuncula MB, Almeida RSC, Specht AM

\begin{tabular}{lc}
\hline Colesterol total aumentado & $59(42,1)$ \\
HDL baixo $(<40 \mathrm{mg} / \mathrm{dL})$ & $8(5,7)$ \\
Tabagistas & $9(6,4)$ \\
Diabéticas & $4(2,9)$ \\
Pouco ativas a sedentárias & $85(60,7)$
\end{tabular}

Nota: Variáveis contínuas expressas como médias \pm desvio padrão; variáveis categóricas expressas como n (\%).

Quando avaliadas as associações entre as variáveis clínicas e a fase reprodutiva, foram encontradas relações estatisticamente significativas no que diz respeito a valores de colesterol total, terapia anti-hipertensiva, aumento no escore total de risco cardiovascular, com valor de $\mathrm{p}<0,05$ para todas as comparações. Ao serem comparadas variáveis clínicas com o RCV aumentado, foram encontradas relações estatisticamente significativas nos testes de pressão arterial sistólica elevada e menopausa, com valor de $\mathrm{p}<0,05$ para ambos (Tabela 2).

Tabela 2 - Associações entre variáveis clínicas. São Leopoldo, 2018.

\begin{tabular}{lc}
\hline $\begin{array}{l}\text { Variável } \mathbf{x} \text { fase do ciclo reprodutivo } \\
\text { (climatério/menopausa) }\end{array}$ & valor $\boldsymbol{p}^{*}$ \\
\hline Colesterol total & $<0,05$ \\
Lipoproteínas de alta densidade & 0,83 \\
Terapia com anti-hipertensivos & $<0,05$ \\
Aumento do Risco Cardiovascular & $<0,01$ \\
& \\
Variável x RCV aumentado & \\
& \\
Tabagismo & 0,01 \\
Pressão Arterial Sistólica elevada & $<0,05$ \\
Menopausa & $<0,05$ \\
\hline
\end{tabular}


Avaliação do risco cardiovascular em mulheres docentes no fim do período reprodutivo 8

Nota: *Valor $\mathrm{p}<0,05$ apresenta significância estatística.

Conforme os dados apresentados na Tabela 2 , foi observada uma relação significativa $(\mathrm{p}<0,05)$ entre os valores do colesterol total e cada fase (climatério e menopausa), indicando que, dependendo da fase do ciclo reprodutivo em que a mulher se encontra, suas taxas de colesterol total poderão sofrer alterações. Em relação ao HDL, não foi observada essa relação $(p=0,83)$.

Em relação à $H A S$, apenas $2,1 \%$ das participantes responderam que apresentavam níveis elevados da PAS como sendo o seu valor pressórico habitual, porém destaca-se o fato de estarem em tratamento. O uso de anti-hipertensivos apresentou uma relação significativa $(\mathrm{p}<0,05)$ quando relacionado à fase do ciclo reprodutivo. Assim, o uso de terapia com anti-hipertensivos está mais presente na fase da menopausa (21 participantes - 23,6\%) quando comparado ao climatério (3 participantes - 5,9\%).

Quanto ao uso do tabaco, os resultados apontaram uma correlação significativa, sendo relacionado o uso com o aumento do $\mathrm{RCV}(\mathrm{p}<0,05)$. Em relação ao $\mathrm{RCV}$, calculado por meio do ERF, apresentam-se os seguintes resultados: a maioria das participantes da amostra obteve risco baixo (116 mulheres - 82,9\%), seguido por risco intermediário $(22-15,7 \%)$ e risco alto $(2-1,4 \%)$.

$\mathrm{Na}$ Tabela 3, podem ser visualizados os resultados do ERF considerando cada fase do ciclo reprodutivo.

Tabela 3 - Escore de risco de Framingham conforme a fase do ciclo reprodutivo. São Leopoldo, 2018.

\begin{tabular}{ccc}
\hline Risco cardiovascular & $\begin{array}{c}\text { Climatério } \\
\mathbf{n}(\mathbf{\%})\end{array}$ & $\begin{array}{c}\text { Menopausa } \\
\mathbf{n}(\mathbf{\%})\end{array}$ \\
\hline Baixo $(<10 \%)$ & $51(100)$ & $65(73,03)$ \\
\hline
\end{tabular}




\begin{tabular}{lcc}
\hline Intermediário (10 a $20 \%)$ & $0(0)$ & $22(24,71)$ \\
Alto $(>20 \%)$ & $0(0)$ & $2(2,25)$ \\
\hline
\end{tabular}

O estudo revelou diferença no grau de risco cardiovascular, de acordo com a fase do ciclo reprodutivo em que estas mulheres referiram estar $(\mathrm{p}<0,05)$, indicando que as mulheres na menopausa apresentam uma relação significativa para o aumento do risco.

\section{Discussão}

O conhecimento precoce dos fatores de risco cardiovascular pode contribuir de maneira expressiva na redução das mortes causadas por DCV. ${ }^{16}$ Diante disto, observaram-se os resultados significativos desse estudo, revelando a forte relação entre as variáveis analisadas na fase da menopausa com o risco cardiovascular aumentado. $\mathrm{Na}$ fase da menopausa as mulheres apresentam modificação no metabolismo lipídico, podendo ocorrer diminuição dos níveis de estrogênio, o que resulta em elevação do perfil lipídico (níveis de colesterol total, lipoproteínas e triglicerídeos) e influencia diretamente no processo da aterosclerose e seus efeitos no organismo. ${ }^{17}$ No presente estudo, os resultados evidenciaram uma taxa expressiva de docentes com níveis elevados do colesterol total, sendo possível observar aumento considerável nesses níveis conforme a fase do ciclo reprodutivo em que as participantes se encontravam.

Ainda que a ausência da prática de exercícios físicos não seja um fator de risco avaliado no ERF, sabe-se que o sedentarismo está fortemente associado à mortalidade por todas as causas e por DCV. Ainda há que se considerar que no sexo feminino o período pós-menopausa parece estar entre os momentos de maior risco para obesidade e sobrepeso. ${ }^{18}$ Ensaio clínico que incluiu 70 mulheres sedentárias e obesas no período da menopausa com idade entre 50 a 79 anos, divididas em um grupo que recebeu treinamento com atividades físicas e outro não treinado, demostrou resultados significativos na redução dos fatores de RCV, reduzindo assim o risco de morte precoce nas mulheres por DCV no grupo intervenção. ${ }^{19}$ Foi predominante o 
Avaliação do risco cardiovascular em mulheres docentes no fim do período reprodutivo | 10

sedentarismo entre a amostra, demonstrando que embora se tenha o conhecimento sobre a importância desta iniciativa na qualidade de vida, encontra-se certa resistência. A relação pode ser fortemente justificada pelo cotidiano corrido das docentes, assim como as demais mulheres inseridas no mercado de trabalho. Estudo que objetivou analisar a associação entre sedentarismo e atividade física com a qualidade de vida de mulheres com idade média de 52,6 $( \pm 4,6)$ anos concluiu que o menor tempo em comportamento sedentário, bem como maiores níveis de atividade física, estão associados à melhor qualidade de vida. ${ }^{20}$

Outro fator que deve ser sinalizado é a associação entre sedentarismo e obesidade, que juntos aumentam o risco de desenvolver as DCV. Conforme conhecimento corrente, a atividade física resultará em benefícios, atenuando o surgimento de doenças crônicas como HAS, DM e dislipidemia, além da redução da gordura corporal, dos níveis de PAS, proporcionando efeitos no controle da glicemia de jejum e nos valores de HDL. ${ }^{18}$ Estudo transversal desenvolvido na China com 8.191 mulheres apresentou prevalência de 13,22\% de DM2. Os autores identificaram aumento de risco de DM2 em mulheres no período pós-menopausa quando comparadas ao período pré-menopausa (OR ajustado $[\mathrm{aOR}]=1,90, \mathrm{IC} 95 \%=1,51-2,37) .{ }^{21}$

Evidenciaram-se baixas taxas de HAS e tabagismo nesta amostra de docentes. Porém, foi observado que há forte relação entre estas, quando presentes, nos resultados finais do ERF. Tais variáveis estão entre os principais fatores de riscos modificáveis que contribuem para o aumento do RCV. ${ }^{18}$ O tabagismo é um importante fator de risco independente para eventos cardiovasculares e mortalidade e, em indivíduos com mais de 60 anos, duplica o RCV, quando comparados a não tabagistas. Ao mesmo tempo, a cessação do hábito nessa faixa etária ainda se mostra relevante na redução de tais riscos, sendo maiores os benefícios à medida que o tempo de cessação aumenta. ${ }^{22}$ Diante das elevadas prevalências de tais fatores de risco e da possibilidade de modificá-los por meio de mudanças comportamentais, é possível inferir a importância do papel do enfermeiro e das políticas públicas neste panorama. 
11 | Santos TT, Barilli SLS, Porciuncula MB, Almeida RSC, Specht AM

O estudo revelou uma diferença significativa entre a média das idades das fases do climatério e da menopausa, fazendo com que, consequentemente, os resultados de risco cardiovascular fossem maiores, segundo o ERF. As mulheres na menopausa apresentaram todos os níveis de risco (baixo - 10\%, intermediário - 10 a $20 \%$ e alto $>20 \%$ ). Por outro lado, o total de mulheres no climatério obteve como resultado o risco baixo.

A percepção de mulheres com maior RCV terem uma prevalência superior na fase da menopausa, devido à idade mais avançada e consequentemente o surgimento de mais eventos cardiovasculares, pode ser concedido às ações do estrogênio no sistema vascular. ${ }^{23}$ Diante dessa declaração, compreende-se que o climatério, por ocorrer anteriormente, é visto como o momento para a implementação de estratégias preventivas no aparecimento de DCV. Reforça-se que o processo de envelhecimento na população feminina resulta em um perfil metabólico alterado, o que interfere na composição e distribuição do tecido adiposo, favorecendo o aumento do peso corporal e refletindo na evolução dos processos ateroscleróticos. ${ }^{16}$

Observou-se predominância do escore de baixo risco $(<10 \%)$, possivelmente devido aos níveis pressóricos adequados apresentados pelas participantes, ao baixo índice de diabéticas e tabagistas, e ainda à média de idade estar abaixo de 60 anos. Tais fatores influenciam na pontuação do escore, repercutindo no resultado final. ${ }^{24}$ Estudo transversal que avaliou o RCV em 102 mulheres nos períodos pré e pós-menopausa, com idade média de $51,5( \pm 7,9)$ anos, concluiu que as mulheres apresentam maior RCV maior no período pós-menopáusico $(1,25 \pm 0,0$

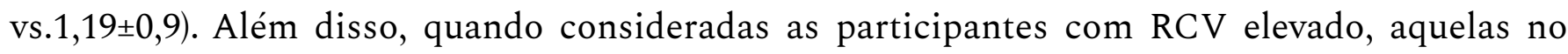
período pós-menopausa apresentaram maior de gordura corporal, colesterol total, glicose e colesterol LDL. ${ }^{25}$

A associação de mais de um fator de risco nas mulheres que apresentaram RCV intermediário e alto foi exposta no estudo. A presença dessa associação de fatores é uma característica do perfil dos indivíduos com o RCV aumentado. ${ }^{18}$ Os presentes resultados, por 
Avaliação do risco cardiovascular em mulheres docentes no fim do período reprodutivo | 12

meio de uma amostra de mulheres docentes, evidenciam a importância de avaliar-se precocemente o RCV nas mulheres que estão no fim do seu período reprodutivo, reiterando a necessidade da atuação preventiva da Enfermagem nesses casos.

\section{Conclusão}

Ao término desta pesquisa, foi possível avaliar o RCV de 140 docentes que estavam no climatério ou menopausa, observando-se o quanto esta fase do ciclo reprodutivo da mulher reflete em alterações de ordem sistêmica. Os resultados trouxeram a relação significativa da menopausa com o aumento do risco cardiovascular, justificando ser mais um fator de risco para a população feminina.

Além de passar por modificações decorrentes da cessação dos hormônios ovarianos neste período, o risco no desenvolvimento de DCV aumenta quando associado a outros fatores apresentados pelo ERF, tais como idade avançada, colesterol total elevado, HAS, DM e tabagismo. O estudo apresentou limitação, também encontrada no ERF, caracterizada pela ausência da avaliação do IMC nessa população, pois a obesidade também é um indicador contribuinte no aumento do RCV.

Pode-se concluir que o ERF é uma ferramenta a ser utilizada para o conhecimento do RCV da população, sendo de fácil manuseio e baixo custo, principalmente na atenção primária, e que a partir dos resultados encontrados possa direcionar ações preventivas. É importante ainda destacar que este escore pode ser aplicado novamente após um período, para que se tenha um retorno dos resultados obtidos, diante da adesão ao tratamento e conhecimento do RCV que cada indivíduo possui, visto que o cálculo é baseado na situação atual do indivíduo.

Atuar na prevenção dos agravos dos RCV, uma vez que as taxas de morbimortalidade crescem de forma expressiva, pode ser entendido como uma estratégia fundamental para a assistência de enfermagem. A implementação de intervenções objetivando melhor qualidade de 
vida das mulheres, não somente nesta fase, mas também ao longo dos anos, trará a conscientização sobre o impacto destas na saúde e as auxiliarão a enfrentar essa fase com menos risco de desenvolver DCV. Diante disso, almeja-se que novas pesquisas continuem sendo praticadas dentro dessa temática, contribuindo assim na disseminação de conhecimentos e novas estratégias para a prevenção e promoção a saúde da população, bem como na redução das taxas de morbidade e mortalidade por DCV.

\section{Referências}

1. Organização Pan-Americana da Saúde. Organização Mundial da Saúde (OPAS/OMS Brasil). Doenças cardiovasculares [Internet]. 2017 [acesso 2018 mar 26]. Disponível em: http://www.paho.org/bra/index.php?option=com_content\&view=article\&id=5253:doencascardiovasculares\&Itemid $=839$

2. Centers for Disease Control and Prevention (CDC). Women and heart disease [Internet]. 2020 [cited 2020 abr 18]. Available from: https://www.cdc.gov/heartdisease/women.htm

3. National Heart, Lung and Blood Institute (NHI). Advancing women heart health [Internet]. 2019 [cited 2020 abr 18]. Available from: https://www.nhlbi.nih.gov/health-topics/all-publications-andresources/advancing-womens-heart-health

4. El Khoudary SR, Thurston RC. Cardiovascular implications of the menopause transition: endogenous sex hormones and vasomotor symptoms. Obstet Gynecol Clin North Am. 2018;45(4):641-61. doi: https://doi.org/10.1016/j.ogc.2018.07.006

5. Newson L. Menopause and cardiovascular disease. Post Reprod Health. 2018;24(1):44-9. doi: https://doi.org/10.1177/2053369117749675

6. Ministério da Saúde (BR). Projeção da população das unidades da federação por sexo e grupos de idade: 2000-2030 [Internet]. 2017 [acesso 2017 set 19]. Disponível em: http://tabnet.datasus.gov.br/cgi/tabcgi.exe?ibge/cnv/projpopuf.def

7. Mühleck F, Laufs U. Primärprävention der koronaren Herzkrankheit. Herz. 2020;45(1):39-49. doi: https://doi.org/10.1007/s00059-020-04901-7

8. Ministério da Saúde (BR). Morbidade hospitalar no Sistema Único de Saúde: óbitos por capítulo CID10 segundo Região/Unidade da Federação no sexo feminino: fevereiro 2010-fevereiro 2020 [Internet]. 2020 [acesso 2020 abr 18]. Disponível em: http://tabnet.datasus.gov.br/cgi/tabcgi.exe?sih/cnv/niuf.def

9. Farias WKS, Rocha TPO, Melo JB, Fonseca EJNC, Fernandes DR, Pontes LP, et al. Evaluation of 
Avaliação do risco cardiovascular em mulheres docentes no fim do período reprodutivo | 14

endothelial function in pre-menopausal women with coronary arterial disease. Int J Cardiovasc Sci. 2017;3(30):227-34. doi: http://dx.doi.org/10.5935/2359-4802.20170043

10. Instituto Brasileiro de Geografia e Estatística (IBGE). Expectativa de vida do brasileiro sobe para 75,8 anos [Internet]. 2017 [acesso 2018 out 20]. Disponível em: https://agenciadenoticias.ibge.gov.br/agenciasala-de-imprensa/2013-agencia-de-noticias/releases/18470-em-2016-expectativa-de-vida-era-de-75-8-anos

11. El Khoudary SR, Santoro N, Chen HY, Tepper PG, Brooks MM, Thurston RC, et al. Trajectories of estradiol and follicle-stimulating hormone over the menopause transition and early markers of atherosclerosis after menopause. Eur J Prev Cardiol. 2016;23:694-703. doi: https://doi.org/10.1177/2047487315607044

12. Lotufo, PA. O escore de risco de Framingham para doenças cardiovasculares. Rev Med. 2008;4(87):232-7. doi: https://doi.org/10.11606/issn.1679-9836.v87i4p232-237

13. Gomes RAF, Barros IML, Ferreira MNL, Costa LOBF. Carotid atherosclerosis in pre- and postmenopausal women with a history of pregnancy-induced hypertension: case-control study. Int J Cardiovasc Sci. 2018;4(31):359-66. doi: http://www.dx.doi.org/10.5935/2359-4802.20180033

14. CONSELHO NACIONAL DE SAÚDE. Resolução no 466, de 12 de dezembro de 2012. Diretrizes e normas regulamentadoras de pesquisas envolvendo seres humanos. Brasília, DF, 2013. Disponível em: http://conselho.saude.gov.br/resolucoes/2012/Reso466.pdf. Acesso em: 26 mar. 2018.

15. CONSELHO NACIONAL DE SAÚDE. Norma Operacional n001/2013. Brasília, DF,2013. Disponível em:

http://andromeda.ensp.fiocruz.br/etica/sites/default/files/documentos/CNS\%20\%20Norma\%20Operacional \%20001.pdf. Acesso em: 18 abr. 2020.

16. Melo JB, Campos RCA, Carvalho PC, Meireles MF, Andrade MVG, Rocha TPO, et al. Cardiovascular risk factors in climacteric women with coronary artery disease. Int J Cardiovasc Sci. 2017;1(31):4-11. doi: http://www.dx.doi.org/10.5935/2359-4802.20170056

17. El Khoudary S, Venugopal V, Manson JE, Brooks MM, Santoro N, Black DM, et al. Heart fat and carotid artery atherosclerosis progression in recently menopausal women. Menopause. 2020;27(3):255-62. doi: 10.1097/GME.0000000000001472

18. Sociedade Brasileira de Cardiologia (SBC). Atualização da diretriz de prevenção cardiovascular da Sociedade Brasileira de Cardiologia - 2019 [Internet]. 2019 [acesso em 2020 abr 18]; Disponível em: http://publicacoes.cardiol.br/2014/diretrizes/2019/atualizacao-da-diretriz-de-prevencao-cardiovascularda-sociedade-brasileira-de-cardiologia-2019.asp

19. Chagas EFB, Bonfim MR, Brondino NCM, Monteiro HL. Exercício físico e fatores de risco cardiovasculares em mulheres obesas na pós-menopausa. Rev Bras Med Esporte. 2015;21(1):65-9. doi: $10.1590 / 1517-86922015210101834$ 
20. Marín-Jiménez N, Ruiz-Montero PJ, De la Flor-Alemany M, Aranda P, Aparicio VA. Association of objectively measured sedentary behavior and physical activity levels with health-related quality of life in middle-aged women. Menopause. 2020;27(4):437-43. doi: 10.1097/GME.0000000000001494

21. Ren Y, Zhang M, Liu Y, Sun X, Wang B, Zhao Y, Liu D, et al. Association of menopause and type 2 diabetes mellitus. Menopause. 2019;26(3):325-30. doi: 10.1097/GME.0000000000001200

22. Gauna AG. En mayores de 60 años el tabaquismo activo duplica la mortalidad cardiovascular y su cesación la reduce. Evid Actuali Práct Ambul [Internet]. 2016 [acceso 2020 abr 18];19(3):84. Disponible en: https://www.fundacionmf.org.ar/files/6ae5688bc43de711b692334f2bb7816f.pdf

23. Carvalho GAC, Reis SAR, Kapitzky SAA, Franco TP, Rocha LLV. Prevalência das doenças cardiovasculares no brasil - um estudo descritivo e retrospectivo. Braz J Surg Clin Res [Internet]. 2016 [acesso 2018 out 20];16(3):12-7. Disponível em: https://www.mastereditora.com.br/periodico/20161104_073021.pdf

24. Sousa NP, Sousa MF, Araújo DER, Santos WS, Lima LR, Rehen TCS. Estratificação do risco cardiovascular na atenção primária segundo escore de Framingham. Tempus (Brasília). 2016;10(1):157-68. doi: http://dx.doi.org/10.18569/tempus.v10i1.1862

25. Dallazen F, Winkelmann ER, Berlezi EM. Cardiovascular risk evaluated by the conicity index in climacteric women: a comparative analysis between the pre and post-menopause periods. Sci Med. 2017;27(4):ID28268. doi: http://dx.doi.org/10.15448/1980-6108.2017.4.28268

\section{Autor correspondente}

Andréia Martins Specht

E-mail: spechtandreia@gmail.com

Endereço: Av. Dr. Nilo Peçanha, 1600 - Boa Vista, Porto Alegre - RS.

CEP: 91330-002

\section{Contribuições de Autoria}

1 - Tamires Tássia dos Santos

Concepção e planejamento do projeto de pesquisa, execução da coleta de dados, análise e interpretação dos dados, redação.

\section{2 - Sofia Louise Santin Barilli}

Interpretação dos dados, redação e revisão crítica. 
Avaliação do risco cardiovascular em mulheres docentes no fim do período reprodutivo | 16

\section{3 - Mariana Bello Porciuncula}

Redação e revisão crítica.

\section{4 - Rafaela dos Santos Charão de Almeida}

Redação e revisão crítica.

\section{5 - Andréia Martins Specht}

Concepção, planejamento e orientação do projeto de pesquisa, análise e interpretação dos dados, redação e revisão crítica.

\section{Como citar este artigo}

Santos TT, Barilli SLS, Porciuncula MB, Almeida RSC, Specht AM. Avaliação do risco cardiovascular em mulheres docentes no fim do período reprodutivo. Rev. Enferm. UFSM. 2020 [Acesso em: Anos Mês Dia]; vol.10 e38: 1-16. DOI:https://doi.org/10.5902/2179769238513 